\title{
Degeneracy between mass and spin in black-hole-binary waveforms
}

\author{
Emily Baird, Stephen Fairhurst, Mark Hannam, and Patricia Murphy \\ School of Physics and Astronomy, Cardiff University, Cardiff CF24 3AA, United Kingdom
}

(Received 12 November 2012; published 22 January 2013)

\begin{abstract}
We explore the degeneracy between mass and spin in gravitational waveforms emitted by blackhole-binary coalescences. We focus on spin-aligned waveforms and obtain our results using phenomenological models that were tuned to numerical-relativity simulations. A degeneracy is known for low-mass binaries (particularly neutron-star binaries), where gravitational-wave detectors are sensitive to only the inspiral phase, and the waveform can be modeled by post-Newtonian theory. Here, we consider black-hole binaries, where detectors will also be sensitive to the merger and ringdown, and demonstrate that the degeneracy persists across a broad mass range. At low masses, the degeneracy is between mass ratio and the black-hole spins, with chirp mass accurately determined. At higher masses, the degeneracy persists but is not so clearly characterized by constant chirp mass as the merger and ringdown become more significant. We consider the importance of this degeneracy both for performing searches (including searches where only nonspinning templates are used) and in parameter extraction from observed systems. We compare observational capabilities between the early $(\sim 2015)$ and final (2018 onwards) versions of the Advanced LIGO detector.
\end{abstract}

DOI: 10.1103/PhysRevD.87.024035

\section{INTRODUCTION}

The Advanced LIGO and Virgo detectors are likely to allow us to observe numerous black-hole-binary coalescences in the coming years [1,2]. While the detectors are being installed, the challenge is to devise search and source extraction methods that will identify all black-hole-binary signals in the data while also allowing for the most accurate estimation of the source parameters possible.

Our understanding of gravitational waveforms emitted by black-hole mergers has improved dramatically over the past five years, with the aid of a large number of numerical simulations, from which several models of the waveforms have been constructed (see e.g., Ref. [3] for an overview). A large fraction of the simulations have focused on nonprecessing systems (where the black holes are either nonspinning or have spins aligned with the orbital angular momentum); see, for example, Refs. [4,5]. Other than for high black-hole spins (greater than $a \sim 0.8$ ), this space has been rather well covered for comparable-mass binaries, and several waveform models are available [6-8]. The models characterize waveforms based on the masses of the two components and, in the case of the models we will consider in this paper, the total spin of the system. We will make use of these waveform models to investigate degeneracies in the waveforms at signal-to-noise ratios (SNRs) close to the detection threshold in advanced detectors. Since we are only considering a three-dimensional subspace of the full eight-dimensional space of binary masses and spins, many effects, most notably precession, cannot be probed. However, recent results have shown that the waveform for a precessing binary can be factorized into precessional effects and a nonprecessional part that is well modeled by the three parameters we consider here [9].
PACS numbers: 04.30.- w, 04.25.dg, 04.70.Bw, 04.80.Nn

Thus, although strong precession may mitigate the degeneracy considered here, it is likely that even precessingbinary signals will share some of the features we observe.

In post-Newtonian (PN) theory, there is a well known degeneracy between the black holes' mass ratio and the black-hole spins, which arises at 1.5PN order, while a combination of the total mass and mass ratio (the "chirp mass") remains relatively well constrained $[10,11]$. Here, our focus is on higher-mass waveforms that include a merger and ringdown. We demonstrate that the degeneracy persists in the full waveforms and also up to higher masses where only the later part of the inspiral and the merger/ ringdown are in the detector's sensitivity band. However, for the high-mass binaries, the degeneracy in mass and spin is not so clearly characterized by the chirp mass.

A degeneracy in the emitted gravitational waveform across the parameter space has numerous consequences for gravitational wave (GW) searches, both good and bad. The positive effect is that a degeneracy in the parameter space will reduce the volume that needs to be searched, thereby reducing the computational cost and trials factor associated with the search. The majority of searches of LIGO and Virgo data have made use of nonspinning components in the template waveforms (see e.g., Refs. [12,13]). A degeneracy between mass and spin means that the template waveforms have covered a larger fraction of the parameter space than might be naively expected. Furthermore, it has recently been argued that a twodimensional bank of templates is sufficient to cover the space of spinning neutron-star binaries [14]. In this paper, we investigate the effect of using nonspinning waveforms in a search for black-hole binaries with spins and show that the mass-spin degeneracy renders the search more sensitive to spinning systems than might be expected. Nevertheless, 
a search using waveforms incorporating spin effects would be a significant improvement and the degeneracy should make it computationally feasible.

On the other hand, a degeneracy has a negative effect on parameter estimation. To make the most of gravitationalwave observations, accurate extraction of the physical parameters is of paramount importance. There are detailed multidimensional methods under development to accurately recover the parameters [15-18]. However, there is nothing that can be done about a real degeneracy in the emitted waveforms - there is no way of telling them apart. We evaluate the effects of mass-spin waveform degeneracy on our ability to accurately recover masses and spins and discuss the astrophysical implications. In the process we introduce a simple method to estimate the parameterestimation confidence intervals.

Throughout the paper, we will provide sample results using a number of waveforms at different masses and mass ratios. We also make use of a number of noise curves for the advanced detectors, to illustrate how this effect is likely to change as the detectors approach their final, design sensitivity. No attempt has been made to perform an exhaustive study across the full parameter space, and this is left as a future project.

The layout of the paper is as follows: in Sec. II we outline the waveform models that we use, and discuss some of the assumptions that went into producing them and their range of applicability. We also discuss the interpretation of results in terms of mismatches between waveforms and introduce the detector noise curves used in our studies. In Sec. III we focus on the degeneracy between mass ratio and spin for low-mass binaries, giving the post-Newtonian argument for this degeneracy and illustrating the degeneracy for a number of cases and different detector sensitivities, and discuss implications for searches. Then, in Sec. IV we extend the results to higher-mass binaries and show that the degeneracy persists, although in a different form. In Sec. V we discuss implications for the accurate estimation of parameters and astrophysical inference.

\section{MODEL AND METHODS}

\section{A. The phenomenological waveform models}

We describe the GW signal from black-hole binaries with nonprecessing spins (i.e., the spins are aligned or antialigned with the binary's orbital angular momentum) using the phenomenological models presented in Refs. [6,7]. For consistency with the labeling used within the LIGO-Virgo Collaboration [19] we refer to these models respectively as "PhenomB" and "PhenomC." ("PhenomA" refers to an earlier model of nonspinning binaries [20-22].) In both models the waveforms are parametrized by their total mass $M=m_{1}+m_{2}$, mass ratio $\eta=m_{1} m_{2} / M^{2}$, and an effective total spin parameter

$$
\chi=\frac{1}{M}\left(m_{1} \chi_{1}+m_{2} \chi_{2}\right)=\chi_{s}+\delta \chi_{a},
$$

where $\chi_{i}=S_{i} / m_{i}^{2}$ for each black hole with angular momentum $S_{i}, \quad \delta=\left(m_{1}-m_{2}\right) / M$, and the symmetric and antisymmetric combinations of the spins are $\chi_{s}=\left(\chi_{1}+\chi_{2}\right) / 2$ and $\chi_{a}=\left(\chi_{1}-\chi_{2}\right) / 2$, respectively. The phenomenological models incorporate a PN description of the inspiral, while the merger and ringdown regimes are tuned using the results of numerical simulations.

Both models have the same basic structure. The waveform is represented in the Fourier domain as $h(f)=$ $A(f) e^{i \Psi(f)}$. The amplitude $A(f)$ and phase $\Psi(f)$ are modeled separately, using input from PN theory (inspiral), the observed properties of NR waveforms (plunge-merger), or results from perturbation theory (ringdown). The models are all power series in the frequency $f$, and the coefficients in the model are written as polynomials in the two physical parameters $\eta$ and $\chi$ (the total mass is an overall scale factor), and it is the coefficients of these polynomials that are then calibrated to hybrids of PN and NR waveforms. For both models, the amplitude is constructed in a similar manner, with expressions for each of the inspiral, plungemerger, and ringdown portions of the waveform modeled independently. In PhenomB [6] the three parts are connected as piecewise functions, and in PhenomC [7] smooth tanh-function interpolation is used. To obtain an expression for the phasing, PhenomB uses a single series expansion, matching the coefficients beyond leading order to hybrid waveforms, as well as results from the test mass $(\eta \rightarrow 0)$ limit. PhenomC, on the other hand, uses the complete TaylorF2 PN inspiral phasing, and only the late inspiral/ merger phase is fitted in a narrow frequency range to numerical simulations, while the ringdown waveform is obtained from analytically derived quasinormal mode expressions for the frequency and attached continuously to the merger phase. There are 54 free parameters in PhenomB, and 45 in PhenomC, although the final models are both functions of only $\{M, \eta, \chi\}$. There are three notable differences between the two models: (i) the PhenomB PN-NR hybrids are produced in the time domain, using TaylorT1 for the PN part, and the PhenomC hybrids are produced in the frequency domain, using TaylorF2 for the PN part [23]; (ii) PhenomB incorporates information about the test-mass limit; (iii) in PhenomC the phase evolution during inspiral incorporates PN calculations up to 3.5PN order (although the spin terms are complete only up to 2.5PN), while in PhenomB only the leading-order PN inspiral term is fixed, and the remaining terms up to 3.5PN order are tuned to the hybrid waveforms.

There is good qualitative agreement between the two models [7], although no detailed quantitative comparison has yet been performed. We choose to work with PhenomB for generating the results presented in this paper. We have also cross-checked some of the calculations against PhenomC, and we comment further on this in Sec. IV. 


\section{B. Detectors and noise curves}

In this paper we compare spinning- and nonspinningbinary signals with reference to the expected sensitivity of the Advanced LIGO detector (aLIGO) [24-26]. The sensitivity curves we use are shown in Fig. 1. During the early science runs, expected around 2015, the Advanced LIGO detector is unlikely to be at its full design sensitivity. Consequently, we use the "early aLIGO" noise curve [27] to give results indicative of what may be achieved in the early runs. At its optimum sensitivity several years later, the anticipated sensitivity is given by the "zerodetuned high-power" noise curve [28]. In this paper we will take that as the "final" design sensitivity of the detector. Over the parameter space of binaries that we study in this paper, the early aLIGO curve represents a sensitivity of roughly 5 times greater in the SNR than in the initial LIGO detectors during their final S6 science run, which corresponds to an increase in potential sources of 2 orders of magnitude. The final curve represents a further factor of 3 improvement in SNR, or about 30 times as many potential sources over early aLIGO. Finally, we also consider the "no signal recycling" (no-SRM) configuration of the detector, that could be achieved by the aLIGO detector operating without a signal recycling cavity. This has comparable low-frequency sensitivity to the final configuration but significantly worse sensitivity at high frequencies. Although it is unlikely to be an observational mode, the non-signal-recycled curve offers a means to compare the effects of low- and high-frequency sensitivity upon our results.

\section{Waveform mismatches}

We use the standard inner product between two waveforms, $h_{1}(f)$ and $h_{2}(f)$ with respect to the power spectral density $S_{n}(f)$ of a detector [29],

$$
\left(h_{1} \mid h_{2}\right)=4 \operatorname{Re} \int_{0}^{\infty} \frac{h_{1}(f) h_{2}^{*}(f)}{S_{n}(f)} d f
$$

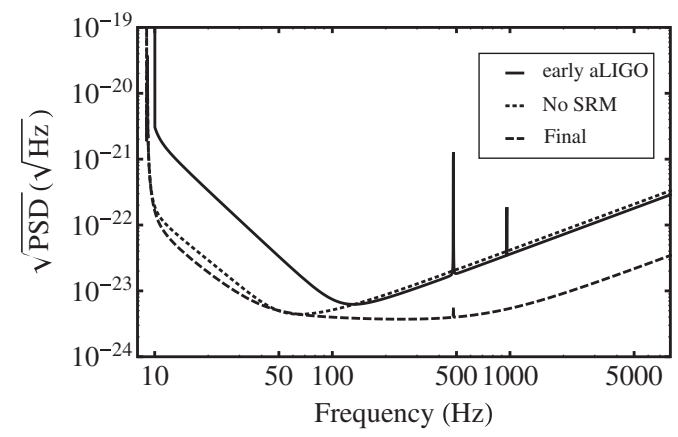

FIG. 1. Noise curves for the Advanced LIGO detector configurations that we consider in this paper: early, no-SRM and final, which corresponds to the zero-detuned, high-power configuration. See text for more details.
The match between two waveforms is defined as their normalized inner product, maximized over time and phase shifts of the waveform:

$$
M\left(h_{1}, h_{2}\right)=\max _{\Delta t, \Delta \phi} \frac{\left(h_{2} \mid h_{2}\right)}{\left|h_{1}\right|\left|h_{2}\right|} .
$$

Alternatively, we can consider the situation of a true waveform produced by a physical source, $h_{T}$, and a model waveform $h_{M}$ that we will use to search for the real signal in detector data. If we normalize both waveforms, then we can split the model waveform into a component parallel to the true waveform, plus a component that is orthogonal to the true waveform, in the sense of our inner product. In other words, we have

$$
\hat{h}_{M}=\sqrt{1-x^{2}} \hat{h}_{T}+x \hat{h}_{E}
$$

where $\hat{h}_{E}$ is the normalized "error" waveform that satisfies $\left(h_{T} \mid h_{E}\right)=0$. In this form, the match is $M\left(h_{T}, h_{M}\right)=$ $\sqrt{1-x^{2}} \approx 1-x^{2} / 2$. Thus, the match is directly related to the (relative) amplitude of the error waveform.

In a GW search, a template bank of model waveforms is constructed [30] such that the match between every point in the waveform parameter space and the nearest template in the bank is at least 0.97. Assuming that the model waveforms are physically correct, such a template bank ensures that we will lose no more than $10 \%$ of signals in our search. (A match of 0.97 means that the sensitivity range of the detector is only $97 \%$ of its optimum, and the detector is therefore sensitive to only $0.97^{3} \approx 0.9$ of its optimum volume, and so we lose about $10 \%$ of signals.)

If the physical waveforms do not agree exactly with the model waveforms, this will lead to an additional loss in match between a signal and the best-matched template, and consequently a reduction in the number of signals observed above threshold. In addition, to counter nonstationary detector noise, a number of signal consistency tests are included into analyses to distinguish signals from nonstationarities or "glitches" in the data [31]. These tests are used to either remove completely any transients that do not match the templates or else to downweight their significance. Since searches performed to date have made use of nonspinning waveform families these thresholds have been set relatively loosely (and tested with spinning signals) to ensure they are not removing signals. For the high matches between signal and template we consider in this paper, the effect of signal consistency tests will be minimal, and we will not consider it further.

In the next sections we will identify the regions of the nonspinning waveform parameter space that provide a match of greater than 0.97 with the chosen waveform (which will usually incorporate spin). In doing so, we densely sample the nonspinning waveforms to identify all points with a match above 0.97 . When performing a search, there will then be two contributions to the 
mismatch between the signal and the best-matched template: one due to the difference between the waveform and search space and a second arising from the discrete sampling of the template space. The match between the signal and the closest template is guaranteed to be above 0.94 as the mismatches add linearly in this case (see e.g., Ref. [32] for details). We are therefore requiring that the potential loss of SNR due to a mismatch between the model waveform and true waveform is no greater than the maximum possible loss due to the discreteness of the template bank.

We will also investigate how the parameters of the best-match nonspinning waveform vary when the signal corresponds to a nonprecessing binary. In Sec. V we will see how the match can be used to give an estimate of the parameter estimation accuracy.

\section{DEGENERACY BETWEEN $\eta$ AND $\chi$ AT LOW MASSES}

We first consider the degeneracy between the symmetric mass ratio $\eta$ and the effective total spin $\chi$ of the binary. This effect is already well known in PN theory [10,11], which we will discuss first, and then we will look at inspiral-merger-ringdown (IMR) signals at three different values of the total mass, $M=\{20,50,100\} M_{\odot}$.

\section{A. Degeneracy in PN theory}

The phase evolution of a compact binary in PN theory has been calculated up to 3.5PN order in the nonspinning terms, and up to 2.5PN in the spin effects; see Ref. [23] for a recent summary of PN treatments of the phase. Up to the leading order that includes spin, the phase for nonprecessing binaries is given in the frequency domain by

$$
\begin{aligned}
\Psi(f)= & \frac{3}{128 \eta v^{5}}\left\{1+v^{2}\left[\frac{3715}{756}+\frac{55 \eta}{9}\right]\right. \\
& \left.-v^{3}\left[16 \pi-\left(\frac{113}{3}-\frac{76 \eta}{3}\right) \chi_{s}-\frac{113 \delta}{3} \chi_{a}\right]\right\},
\end{aligned}
$$

where $v=(\pi M f)^{1 / 3}$. If we define the chirp mass of the binary as $\mathcal{M}=M \eta^{3 / 5}$, then we see that the leading factor is proportional to $1 /(\mathcal{M} \pi f)^{5 / 3}$, and the phase evolution is dominated by the chirp mass. This motivates the observation that in GW searches we expect to measure the chirp mass with high accuracy, and we will see examples of this in Sec. III B. At the next-to-leading order, the phase evolution depends on the mass ratio $\eta$, and dependence on the spins enters at the following order. We can absorb all of the spin effects at this order into an effective spin term, $\chi_{\mathrm{PN}}=\chi_{s}+\delta \chi_{a}-(76 \eta / 113) \chi_{s}$, and this is what is proposed in the inspiral template family discussed in Ref. [33] (and this is proportional to the leading-order spin-orbit parameter $\beta$ used in Refs. [10,11]). The phenomenological models [6,7] use the simpler effective spin $\chi=\chi_{s}+\delta \chi_{a}$ to describe full inspiral, merger and ringdown waveforms.
If we adopt for the moment the PN effective spin term, we can write the deviation from the leading-order term as

$$
\Delta \Psi(f)=\frac{3}{128 \eta v^{3}}\left[\frac{3715}{756}+\frac{55 \eta}{9}+v\left(\frac{113 \chi_{\mathrm{PN}}}{3}-16 \pi\right)\right] .
$$

Thus, it is possible to mimic the effect of spin by modifying the mass ratio (while keeping the chirp mass constant). It is clear, however, that the required modification to $\eta$ will vary as $v$ changes over the course of the chirp, as expected as this is only an approximate degeneracy. The majority of the power from a binary merger is accumulated between around $30-300 \mathrm{~Hz}$, and over this range $\nu$ changes by only a factor of 2. This explains why the approximation is reasonable across the whole signal, and this leads to the common claim that nonspinning templates can be used to detect GW signals from spinning binaries, but there will be an offset in the measurement of the mass ratio and the total mass. It may also be possible to exploit this degeneracy to search for spinning binaries using a nonspinning model, but with $\eta$ extended to unphysical values, $\eta>0.25$, in order to cover more of the spinning-binary parameter space; a similar idea has already been suggested to extend inspiral-only searches beyond their expected region of validity [34]. It is the degeneracy between mass ratio and spin that we will investigate here.

\section{B. Degeneracy in full IMR models}

We would now like to investigate whether the degeneracy from post-Newtonian theory is present in full IMR waveform models.

We consider a set of spinning signals and those nonspinning waveforms (i.e., the phenomenological model with $\chi=0$ ) that provide a good match to the signal. The efficacy of a waveform model in a GW search can be estimated by calculating the best match (fitting factor) between any member of the waveform model and the target signal. Therefore, this will give an immediate indication of the merits (or otherwise) of using a nonspinning waveform model to search for mergers of spinning black holes. We consider fitting factors above 0.97 to be adequate as discussed previously.

Figure 2 shows the results for a binary with a total mass of $20 M_{\odot}$ and mass ratio $1: 4(\eta=0.16)$, i.e., a $4 M_{\odot}-16 M_{\odot}$ binary, and matches calculated using the early aLIGO spectrum. We consider sources with an effective total spin in the range $[-1,1]$ in steps of 0.1 . For each configuration, we identify nonspinning waveforms which give a match of greater than 0.97 with the spinning source. When the binary spin is zero, the nonspinning model matches the target signal at the correct parameters (indicated by a star), and along a strip in the parameter space with a width of 5\% in mass, and $10 \%$ in $\eta$. When the binary contains spinning black holes, the nonspinning model matches the signal, but with a bias in the mass and mass ratio. The binary spins are 


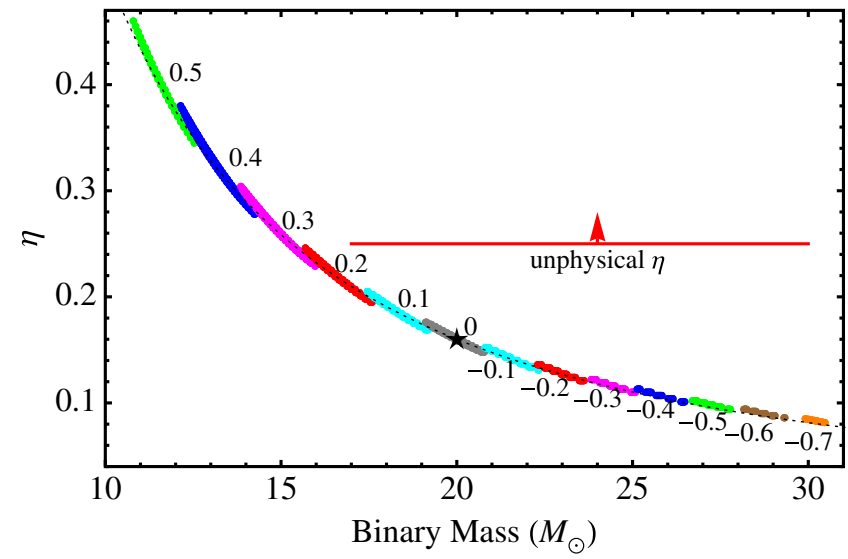

FIG. 2 (color online). Match between spinning $20 M_{\odot}$ waveforms with nonspinning templates in early aLIGO. Each of the strips shows the region of $M-\eta$ space for which the nonspinning waveform has a match of 0.97 or higher with a spinning signal. The effective total spin is indicated next to each region. For spins above 0.5 or below -0.7 the best overlap with a nonspinning waveform is less than 0.97 . The curve of constant chirp mass is indicated by a dotted line.

indicated with different colors, labeled by the effective total spin of the binary. For large-spin signals there are no nonspinning waveforms that have a match above 0.97 . When using the early aLIGO noise curve, the range of spins for which nonspinning waveforms have a match above 0.97 is $[-0.7,0.5]$. However, for spins above about $\chi=0.3$, the best-match waveform has an unphysical value of the mass ratio $\eta$. The curve of constant chirp mass $M \eta^{3 / 5}$ is indicated by a dotted line.

The first thing to note is that, in all cases, the chirp mass of the best-matched nonspinning waveform is very similar to the true chirp mass of the system. We can see from Fig. 2 that the high-match regions follow a line of constant chirp mass. In Fig. 3 we reparameterize the results of Fig. 2 in

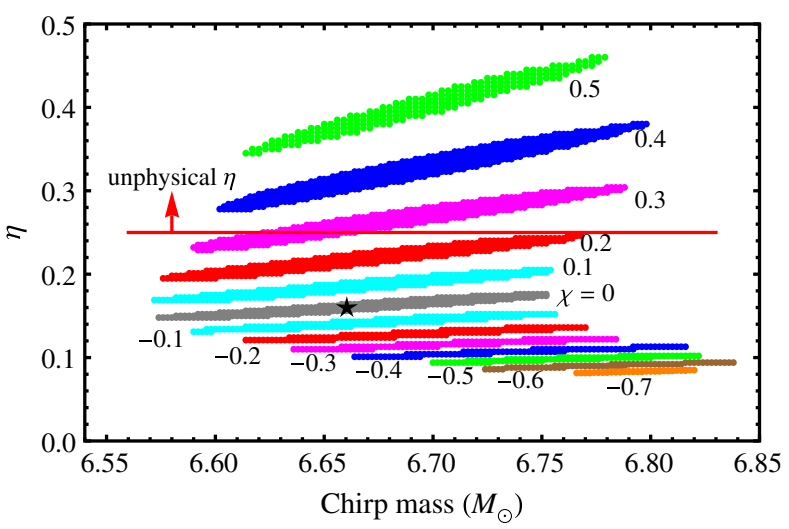

FIG. 3 (color online). The same results as in Fig. 2, but now parametrized in terms of chirp mass and symmetric mass ratio. This figure reinforces the accuracy with which the chirp mass can be recovered. terms of $\eta$ and $\mathcal{M}$. We see that, even though there is a strong degeneracy between $\eta$ and $\chi$, the correct chirp mass $\mathcal{M}=6.66 M_{\odot}$ falls within the high-match region for most values of spin. The deviation from the correct chirp mass is no more than $\sim 3 \%$ for large antialigned spins.

The second important point relates to the mass-spin degeneracy. For aligned-spin binaries $(\chi>0)$, the best match is obtained with a lower-mass, higher-mass-ratio model, while for antialigned spins, the best-matched nonspinning waveform has a higher mass and lower mass ratio. This is to be expected as aligned spins are expected to cause the system to "hang up" and chirp more slowly, mimicking a lower-mass signal.

We caution the reader against overinterpreting the sizes of the high-match regions. The figure does not show the values of the matches, only those that are above 0.97 , and we do not believe that our current waveform models are sufficiently accurate that we can gain significant physical insight from the comparative rate of decay of the matches between different configurations.

It is informative to return to the post-Newtonian phasing given in Eq. (5) to see whether the observed degeneracy matches what is theoretically expected. In low-mass binaries, the signal is dominated by the inspiral, which can be represented in a PN expansion, as described in Sec. III A. The chirp mass is determined to high accuracy by the leading-order term in the PN expansion of the phase in the Fourier domain, and we can then solve Eq. (5) (with fixed chirp mass) to find the symmetric mass ratio that mimics the effect of the spin. We do this by solving (5) as a function of frequency (in the detector's sensitive band) and then averaging over the values of $\eta$ that we obtain. Figure 4 shows the symmetric mass ratio that corresponds to each value of the spin, as predicted from Eq. (5), and as found in the mismatch analysis of the phenomenological models

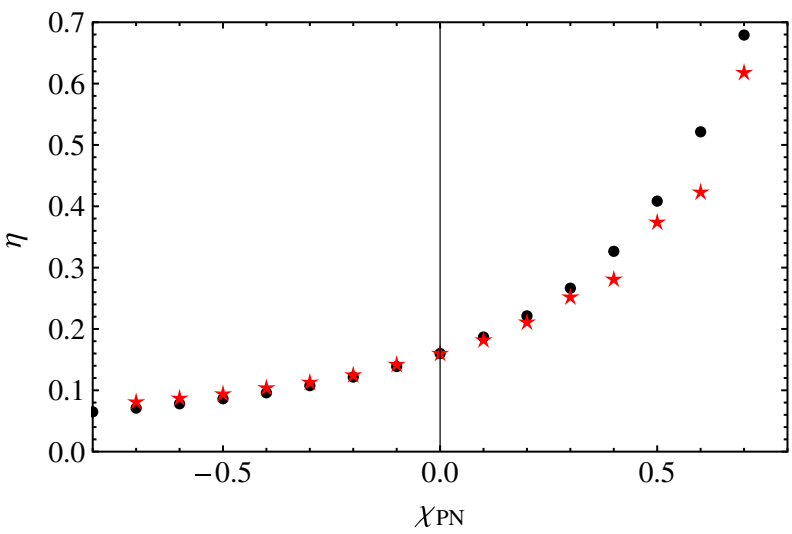

FIG. 4 (color online). Comparison of the $\chi-\eta$ degeneracy as found for the phenomenological model (stars), and from the requirement that $\Delta \Psi=\Delta \Psi\left(\chi_{\mathrm{PN}}=0\right)$ in Eq. (5) for fixed chirp mass, for signals with $M=20 M_{\odot}$ and $\eta=0.16$. The naive PN prediction agrees well with the full IMR results for low spins, and for most antialigned spins. 
that is shown in Fig. 2. We see that the PN estimate is remarkably close to that found for the full IMR models, at least for the $20 M_{\odot}$ binaries used in this example.

\section{Evolution of noise curves}

We present results for two design aLIGO configurations in Fig. 5: the no-signal-recycling (no-SRM) configuration and the final high-power zero-detuned sensitivity curve (final). Qualitatively, the results are quite similar for the no-SRM and final noise curves. Again spinning signals are recovered with a match above 0.97 using nonspinning templates, with a decrease in recovered mass for positive spin systems. The chirp mass is still well recovered. However, the detector's extra sensitivity makes it easier to distinguish the spinning signals with a nonspinning model. The range of spins for which we can find a nonspinning signal with matches greater than 0.97 is now only $\chi \in[-0.4,0.3]$ for no-SRM and $\chi \in[-0.3,0.3]$ for the final configuration. We also see that each match region shrinks, although its location is unchanged. In moving from the early noise curve to no-SRM, the most significant sensitivity improvement is at low frequency, while the final configuration offers much greater high-frequency sensitivity. The results for these two curves are quite comparable
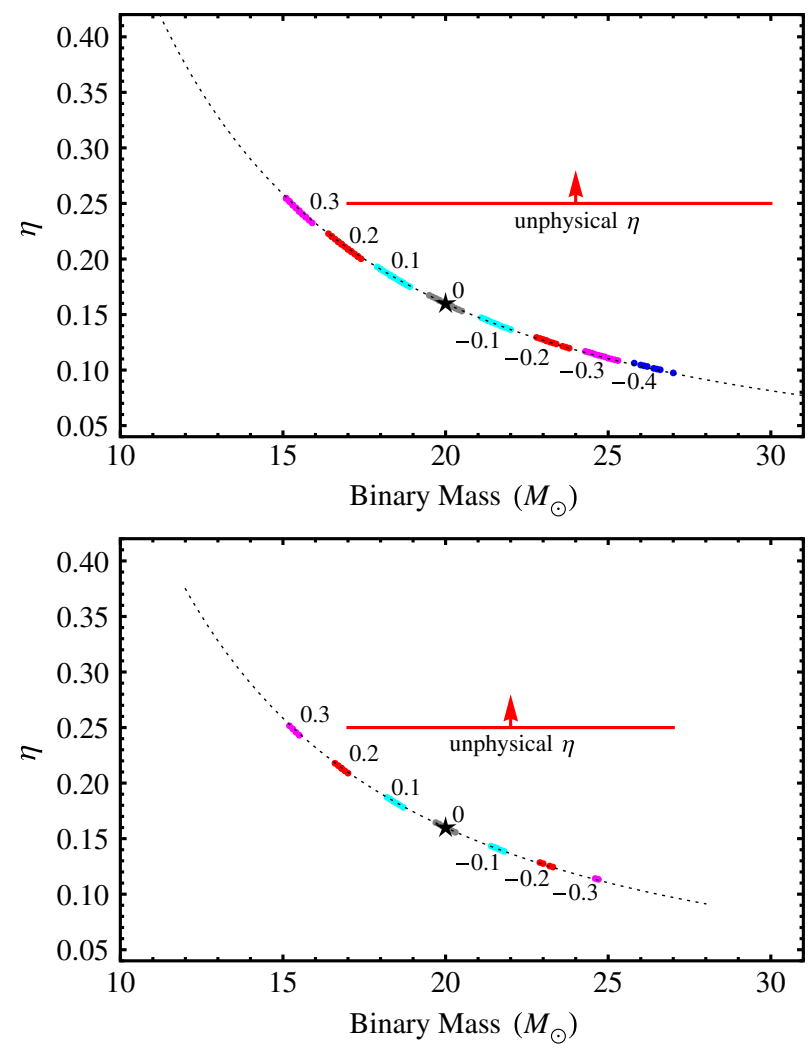

FIG. 5 (color online). Matches of spinning $20 M_{\odot}$ waveforms with nonspinning templates in aLIGO at design sensitivity. The upper panel shows the results for the no-SRM configuration, while the lower panel shows results for the final sensitivity.

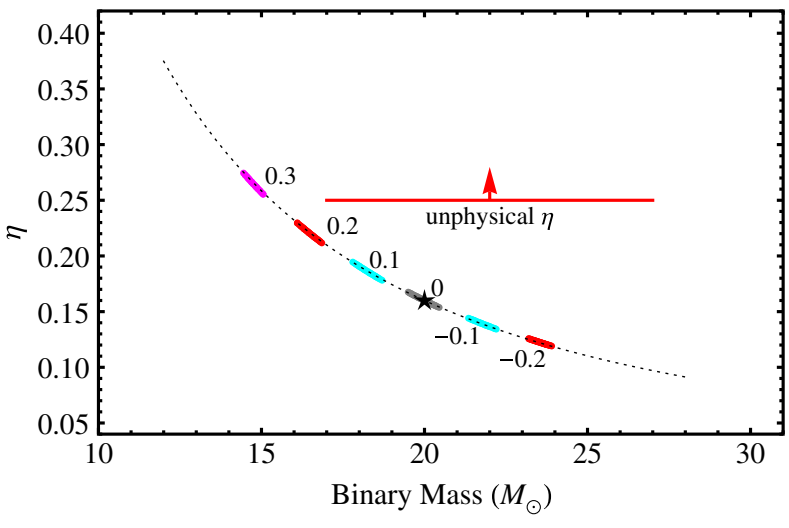

FIG. 6 (color online). Final sensitivity results using an inspiral-only model.

(and significantly better than the early curve) indicating that it is low-frequency sensitivity that provides the biggest improvement in breaking the degeneracy. With the later noise curves, the variation of chirp mass between the spinning signal and nonspinning template is less than $1 \%{ }^{1}$

In the $20 M_{\odot}$ configurations we have studied, most of the SNR is accumulated during the inspiral. For comparison, in Fig. 6 we show the high-power zero-detuned noise curve results when using an inspiral-only waveform model, TaylorF2 $[10,35,36]$, for which the inspiral is modeled to the highest-known post-Newtonian orders (3.5PN in nonspinning terms, and 2.5PN in spin terms). The results are similar to those with the inspiral-merger-ringdown model, but the confidence regions are larger. This suggests that the inclusion of merger and ringdown mitigates to some degree the degeneracy.

\section{MASS-SPIN DEGENERACY AT HIGHER MASSES}

For black-hole binaries with masses greater than $20 M_{\odot}$ the merger and ringdown parts of the signal become increasingly important and contribute an ever increasing fraction of the signal-to-noise ratio. At higher masses, we do not expect the chirp mass to determine the waveform to such an extent as for the $20 M_{\odot}$ system and there is no a priori reason to expect that a degeneracy between mass and spin will persist.

We begin by considering a $50 M_{\odot}$ binary with mass ratio 1:4. At this mass, the merger and ringdown will provide a significant fraction of the signal to noise ratio. As a crude

\footnotetext{
${ }^{1}$ The figures may seem to indicate that a $20 M_{\odot}$ binary with total spin of 0.5 that would be detected by a nonspinning search in the early aLIGO configuration, but not later ones. This is of course not true: the final detector is roughly 3 times more sensitive at these masses, and so a signal with SNR 10 in early aLIGO would have an SNR of 30 in the final configuration. Even with a match of 0.9 , this would still give an SNR of 27, and the match would be sufficient that it would pass any signal consistency tests.
} 
estimate of the effect, imagine that the inspiral part of the waveform is valid up to the innermost stable circular orbit of a point particle orbiting a Schwarzschild black hole, which is at $90 \mathrm{~Hz}$ for a $50 M_{\odot}$ binary. For the early aLIGO noise curve, the inclusion of the merger and ringdown will increase the SNR by a factor of 2. At the final aLIGO sensitivity, the improved low-frequency response increases the contribution of the inspiral but the inclusion of merger and ringdown still increase the SNR by $\sim 20 \%$. Thus, there is no reason to expect that the chirp mass is still well recovered or that the degeneracy discussed previously still holds.

In Fig. 7 we again show the regions of the nonspinning parameter space which give a match greater than 0.97 with a spinning binary (with values of $\chi$ from -1 to 1 in steps of 0.1 ). Here, we show the results for both the early and final aLIGO spectra. The results are qualitatively similar to the $20 M_{\odot}$ binary with spinning signals well recovered by the nonspinning waveforms, and the chirp mass accurately recovered. The sizes of the regions are roughly consistent with the lower mass system, with a mass accuracy of $\sim 10 \%$ and range in $\eta$ of $0.05-0.1$ for the early noise curve. For both early and design curves, signals with spins between 0.2 and -0.4 have matches above 0.97 with nonspinning waveforms. Interestingly the degeneracy still roughly follows the line of constant chirp mass, even though the merger and ringdown contributed significantly to the SNR of the signal.

Next, we increase the mass to $100 M_{\odot}$ and repeat the analysis. At this mass, the point-particle innermost stable circular orbit is at $40 \mathrm{~Hz}$, so there is essentially no power from the inspiral in the initial detectors, and only a small amount in the early aLIGO. Even at the final sensitivity, the inclusion of merger and ringdown increase the SNR by about $60 \%$ over inspiral alone. Thus one expects that it is really the merger that dictates the waveform as seen by the

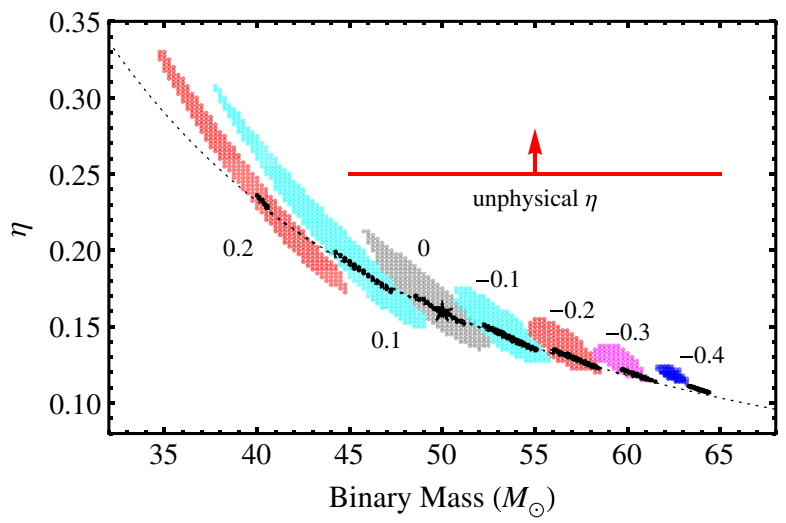

FIG. 7 (color online). Matches of spinning $50 M_{\odot}$ waveforms of mass ratio 4:1 with nonspinning templates in early and final aLIGO. Each of the strips shows the region of $M-\eta$ space for which the nonspinning waveform has a match of 0.97 or higher with a spinning signal.

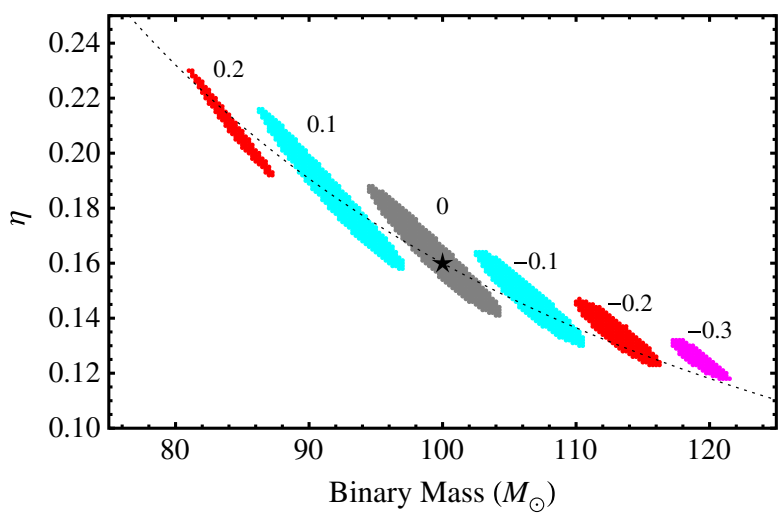

FIG. 8 (color online). Matches of spinning $100 M_{\odot}$ waveforms of mass ratio 4:1 with nonspinning templates in design aLIGO.

detector. Figure 8 shows results for $100 M_{\odot}$, again with $\eta=0.16$. For aLIGO at final design sensitivity, the curves are again lying pretty much along the line of constant chirp mass.

We do not show results for the early aLIGO sensitivity curve in Fig. 8, because the results depend strongly on the choice of model, either PhenomB or PhenomC. The variation of the two models with respect to the physical parameters is sufficiently large through merger and ringdown that they lead to qualitatively different results in our mismatch studies, when we use the early aLIGO noise curve, where the merger and ringdown contribute essentially all of the SNR. The two models were developed as pioneering models of aligned-spin IMR waveforms for use in searches, and for this we expect that they are sufficient; but their fidelity with respect to parameter estimation at high masses has not yet been tested and should be a focus of future work to refine them.

\section{PARAMETER RECOVERY}

Once a black-hole merger has been detected, we wish to extract the signal parameters as accurately as possible. Parameter estimation proceeds by identifying regions of parameter space which give a signal that is most consistent with the data. It stands to reason that these regions will contain waveforms that have a good match with the observed signal. Thus we might expect that confidence regions in parameter estimation are associated with regions of high match between signal and template. In this section, we show this expectation to hold in detail in the high-SNR limit and derive an expression for the value of the match that corresponds to a given confidence at a given SNR.

We begin by presenting the argument, using the Fisher matrix formulation, and then we use this connection to reinterpret our earlier results in terms of parameter recovery. We follow the formalism used Ref. [37], but provide only a brief discussion of the Fisher matrix formalism, and refer the reader to Ref. [37] (and references therein) for more details. 


\section{A. Connection between mismatch and confidence regions}

Let us assume that a signal $h_{0}$ is present in the data. The detector data is given as

$$
s(t)=h_{0}(t)+n(t) .
$$

To investigate signal recovery and parameter extraction at leading order, we Taylor expand the signal in a region of the true parameters $(\theta=0)$ as

$$
h(\theta)=h_{0}+\theta^{i} h_{i}+\cdots .
$$

Here $h_{i}=\partial_{i} h$ is used to denote the derivative of the waveform with respect to the parameters $\theta_{i}$, which will include $M, \eta, \chi$ as well as the amplitude, phase and coalescence time that are maximized over in the match calculations.

The likelihood, for a given set of $\theta_{i}$, is

$$
p(s \mid \theta) \propto \exp \left\{-\frac{(s-h(\theta) \mid s-h(\theta))}{2}\right\} .
$$

Substituting the expressions for $s(6)$ and $h(\theta)$ (7) into the above and keeping leading order terms gives

$$
p(s \mid \theta) \propto \exp \left\{-\frac{(n \mid n)}{2}+\theta_{i}\left(n \mid h_{i}\right)-\frac{\theta_{i} \theta_{j}\left(h_{i} \mid h_{j}\right)}{2}\right\} .
$$

In the context of Bayesian parameter estimation, this can be recast in terms of a posterior probability distribution for the parameters $\theta_{i}$ using Bayes theorem:

$$
p(\theta \mid s) \propto p(s \mid \theta) p(\theta),
$$

where $p(\theta)$ is the prior probability distribution for the parameters. In what follows, we use a uniform prior on the parameters. In general, such a prior will not be physically motivated but, for a detectable signal, the likelihood will be peaked in a small enough region of parameter space to make this approximation reasonable.

Given the above, we are interested in calculating the expected offset between the true parameters and the mean value from the posterior distribution. We also want to evaluate the size of a confidence region containing a given fraction $p$ of the posterior probability. These quantities give us two different measures of the expected accuracy of parameter recovery.

We begin by calculating the mean of the parameter $\theta_{i}$ as

$$
\left\langle\theta_{i}\right\rangle=\int d \theta \theta_{i} p(\theta \mid s)=\left(h_{i} \mid h_{j}\right)^{-1}\left(n \mid h_{j}\right) .
$$

Thus, the mean of the posterior distribution will be offset from the true parameter values due to the presence of noise. One way to characterize this is the expected size of the error waveform, $h_{E}=h\left(\left\langle\theta_{i}\right\rangle\right)-h_{0}$, as

$$
\left\langle h_{E}^{2}\right\rangle_{n}=\left\langle\left(n \mid h_{i}\right)\left(h_{i} \mid h_{j}\right)\left(h_{j} \mid n\right)\right\rangle_{n}=k,
$$

where $k$ is the dimension of the parameter space and \langle\rangle$_{n}$ indicates the expectation value over many noise realizations. Thus, on average the difference between the true signal and "best fit" waveform will have an amplitude of $\sqrt{k}$.

Next, we turn our attention to confidence regions in parameter space-a region $\Theta$ of parameter space that contains a given probability $p$ of the posterior distribution,

$$
p=\int_{\Theta} d \theta p(\theta \mid s) .
$$

There are many ways to construct such a region, and one typically also requires the smallest possible region. To calculate confidence regions in the Fisher approximation, we begin by observing that the covariance between parameters is given as

$$
\left\langle\theta_{i} \theta_{j}\right\rangle=\left(h_{i} \mid h_{j}\right)^{-1} .
$$

Using this expression and Eq. (11), we can reexpress the posterior distribution as

$$
\begin{aligned}
p(\theta \mid s) & \propto \exp \left\{-\frac{1}{2}\left(\theta_{i}-\left\langle\theta_{i}\right\rangle\right)\left(h_{i} \mid h_{j}\right)\left(\theta_{j}-\left\langle\theta_{j}\right\rangle\right)\right\} \\
& \simeq \exp \left\{-\frac{1}{2}\left(|h(\theta)-h(\langle\theta\rangle)|^{2}\right)\right\} .
\end{aligned}
$$

Then, the minimum volume region which contains a fraction $p$ of the posterior probability is the one for which

$$
|h(\theta)-h(\langle\theta\rangle)|^{2}<\chi_{k}^{2}(1-p),
$$

where $\chi_{k}^{2}(1-p)$ is the chi-square value for which there is $1-p$ probability of obtaining that value or larger, and $k$ denotes the degrees of freedom, determined by the number parameters included in $\theta_{i}$. At leading order, the confidence interval contains all points for which the amplitude of the difference between the model and best fit waveforms lies below the given threshold.

There are six parameters in the aligned-spin waveform model: $M, \eta, \chi, A, \phi_{c}$ and $t_{c}$. When calculating matches, we have maximized over the latter three parameters (amplitude, phase and time) and reported match over the three-dimensional space of mass, mass ratio and spin. Thus, we have calculated the three-dimensional matches $M\left(h_{1} \mid h_{2}\right)$, maximized over A, $\phi_{c}$ and $t_{c}$. It is straightforward to recast our earlier results in terms of mismatches. To do this, we note that

$$
\min _{A_{2}}\left|h_{1}-h_{2}\right|^{2}=\left|h_{1}\right|^{2}\left\{1-\frac{\left(h_{1} \mid h_{2}\right)^{2}}{\left|h_{1}\right|^{2}\left|h_{2}\right|^{2}}\right\} .
$$

When we restrict to the subspace of masses and spins, we can write the final term as the match between the waveforms.

By substituting Eq. (17) into Eq. (16), we see that the confidence region is defined by all points in the parameter space for which the match satisfies 


$$
M\left(h(\theta), h(\langle\theta\rangle) \geq 1-\frac{\chi_{k}^{2}(1-p)}{2 \rho^{2}},\right.
$$

where the value of $k$ is given by the dimension of the remaining parameter space. This expression gives us a straightforward way to reinterpret the match calculations presented earlier. For example, with a three-dimensional parameter space, the $90 \%$ confidence region at a given SNR is given by

$$
M\left(h(\theta), h(\langle\theta\rangle) \geq 1-\frac{3.12}{\rho^{2}},\right.
$$

which has the nice property that a match of 0.97 corresponds to a $90 \%$ confidence region at an SNR of 10. For a two-dimensional parameter space, the right-hand side is $1-2.3 / \rho^{2}$, meaning that a match of 0.97 corresponds to 90\% confidence region at an SNR of 9.

We should note that there are numerous assumptions used in the derivation of these results. Most significantly, all of the Fisher matrix results consider only leading-order effects and become less reliable at lower SNR; see e.g., Ref. [37] for a detailed discussion of the issues. When actually calculating confidence regions for a signal, detailed parameter estimation analyses calculate the posterior distribution (15) and integrate to find the region containing $90 \%$ of the probability. Here, we are using a hybrid approach: we use the result of the Fisher matrix calculation to decide the threshold on match required to define the given confidence region, but then calculate the match between waveforms exactly, without recourse to any approximations. Furthermore, we are maximizing the match over three dimensions, analytically for $A$ and $\phi$ and using a Fourier transform to search over time. Thus, we are only applying the Fisher result to the threedimensional subspace of mass, mass ratio and spin. Even there, we are merely using the calculation to determine the appropriate match threshold: the matches are calculated exactly. Consequently, the match regions should be in good agreement with the $90 \%$ confidence regions. We verified that for an SNR of 20, the region identified by our match criteria did contain $90 \%$ of the probability to within $\pm 0.5 \%$.

\section{B. Implications for detectability and parameter estimation}

Let us now return to the results of Secs. III and IV and use the relationship derived above to reinterpret the results in terms of confidence intervals. We can interpret Fig. 2 in two ways: in the context of either a nonspinning or spinaligned templated search. If we were to perform a search with nonspinning templates and observe a $M=20 M_{\odot}$, $\eta=0.16$ binary with nonspinning components with SNR 9 , then the $90 \%$ confidence region would lie along a strip in the parameter space with a width of 5\% in mass, and $10 \%$ in $\eta$. For a binary containing the same mass black holes with spins of -0.5 , the $90 \%$ confidence region would be of approximately the same size (5\% in mass, and $10 \%$ in $\eta$ ) but centered at $M=27 M_{\odot}, \eta=0.10 .^{2}$ The same holds for other values of the spins: the reported statistical uncertainty in parameters is relatively small, while the systematic errors can be significant.

Alternatively, we can consider the three-dimensional space of $M-\eta-\chi$. In that case, a match above 0.97 corresponds to a $90 \%$ confidence region at SNR of 10 . Thus, for example, Fig. 2 shows that the $90 \%$ confidence region for a signal with $m_{1}=16, m_{2}=4$ and $\chi \in[-0.7,0.5]$ would contain waveforms with nonspinning components. Figures 3-8 can be interpreted in a similar manner. For higher SNRs, the confidence intervals shrink. At SNR 10 they correspond to matches of 0.97, SNR 20 to 0.992 and SNR 30 to 0.9965 .

We can use the analysis of the previous section to estimate the $90 \%$ confidence intervals if we employ an aligned-spin model for parameter estimation. For signals with an SNR of 10, the three-dimensional confidence region in $(M, \eta, \chi)$ will correspond to matches greater than 0.97. In these cases we calculate the matches between a given aligned-spin signal, and all other aligned-spin model waveforms with varying mass, mass ratio and total spin. In Fig. 9 we show the 0.97 match volume for a $20 M_{\odot}$ 1:4 binary with $\chi=0.2$, using the final aLIGO noise curve. The top panel shows the full three-dimensional confidence region, and in the lower panels the results are projections onto the $M-\chi$ and $m_{1}-m_{2}$ planes, to aid the interpretation. Figure 10 shows similar results, but for a binary with $\chi=-0.2$.

Since our waveform model now includes spin, the best match is unity at the correct parameters. But from the figures we see that the $90 \%$ confidence region extends well beyond the correct parameters and is far from the naive image conjured by the term "error ellipse." We note in particular that this volume includes a region of the nonspinning binary parameter space; the intersection with the $\chi=0$ plane is consistent with the lower panel of Fig 5. If we were to estimate the parameters of this signal with an aligned-spin waveform model, we would find that the best-fit parameters indicated that the black holes were spinning, but could not rule out that they might in fact be nonspinning — or indeed have spins with the opposite orientation.

The reason for this large uncertainty in the parameters is that the SNR is low; it is only 10 . For an SNR of 20, the 90\% confidence interval corresponds to a match of above 0.992, and for an SNR of 30, the match must be above 0.9965. We see that for these higher SNRs, the confidence

\footnotetext{
${ }^{2}$ Strictly speaking, if the best match between the signal and a model waveform is less than unity, the relation between match and confidence region in Eq. (18) must be modified to reflect this. The $90 \%$ confidence region will contain all points with a match of 0.97 or greater with the best-fit model waveform.
} 

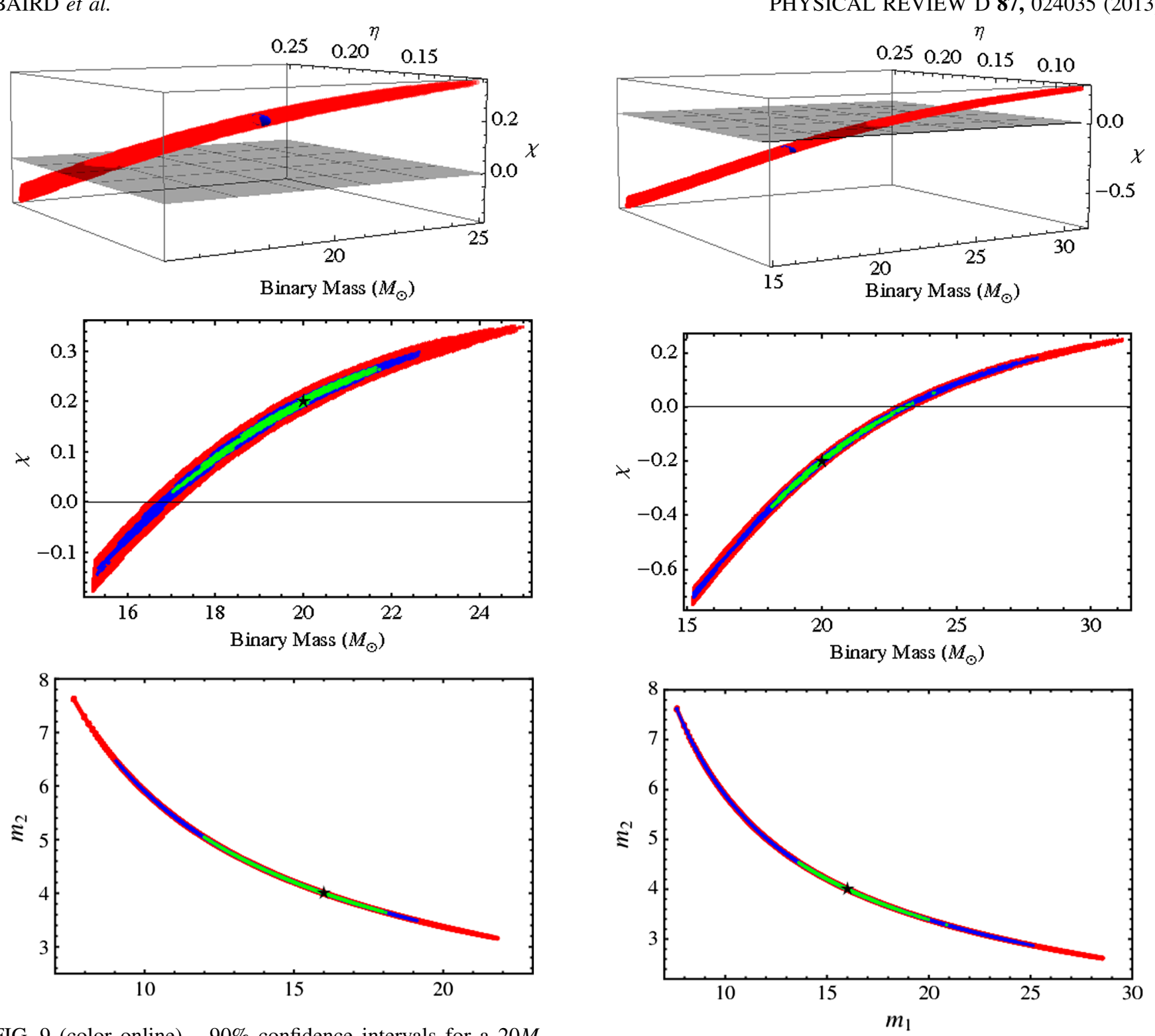

FIG. 9 (color online). $90 \%$ confidence intervals for a $20 M_{\odot}$, $1: 4 \quad(\eta=0.16)$ signal with spin $\chi=0.2$, using the design aLIGO noise curve. The top panel shows the full threedimensional confidence region for $\mathrm{SNR}=10$; the gray surface indicates the $\chi=0$ plane and corresponds to the $\chi=0.2$ region in Fig. 5. The lower two panels show the same data projected onto the $M-\chi$ and $m_{1}-m_{2}$ planes, and also indicate the $90 \%$ confidence regions for SNRs 20 (blue) and 30 (green) (The regions of SNR 10, 20 and 30 are successively smaller). The true physical parameters are indicated by a ball (top panel) or star (lower panels). Regions with unphysical $\eta(>0.25)$ are not shown.

region shrinks. However, only at SNR 30 is a nonspinning signal excluded from the $90 \%$ confidence region for the $\chi=0.2$ case, and even at this SNR (which may be quite rare in aLIGO observations), the smaller mass is determined to within only $25 \%$. When $\chi=-0.2$ (Fig. 10), the $90 \%$ confidence region includes spins as high as $\chi=-0.7$, even when the SNR is 20 . Note also that in these figures we have included only those portions of the

FIG. 10 (color online). The same as Fig. 9, but now the signal has spin $\chi=-0.2$.

confidence regions with $\eta \leq 0.25$, i.e., physically acceptable values; the confidence region would extend further in the upper two panels if the model were not constrained.

\section{CONCLUSIONS}

We have investigated the degeneracy between mass ratio and spin in gravitational waveforms, going beyond inspiral models to include merger and ringdown signals from black-hole-binary mergers. We have used phenomenological IMR models to study the subset of the full binary parameter space that includes nonspinning black holes and black holes with spins parallel or antiparallel to the orbital angular momentum of the binary.

A degeneracy between mass ratio and spin is already known for inspiral signals, which are relevant to 
ground-based gravitational-wave detectors for masses $M \leq 12 M_{\odot}[14,38]$. We find that this degeneracy persists at higher masses, where the detectors are also sensitive to the merger and ringdown. This means that in a GW search that uses only nonspinning binary templates (and this is computationally cheaper than including spin), the signal may still be detected, but the best-match template will have strongly biased parameters. The mass-ratio-spin degeneracy follows lines of constant chirp mass, so the chirp mass will be recovered with reasonable accuracy, even up to high masses and for spinning signals. However, the total mass and mass ratio of the best-matched template will be biased. As shown in Fig. 2, binaries with higher aligned spins will be recovered with a higher mass ratio, while those with antialigned spins will be recovered with a lower mass ratio. If we restrict the search to physical values of the symmetric mass ratio $\eta$, then comparablemass binaries whose spins are aligned with the orbital angular momentum will tend to be missed in the search. We show how these results will change as the detector evolves towards its final sensitivity in Fig. 5 and for higher masses in Figs. 7 and 8.

We also demonstrated that it is possible to use match calculations to estimate the confidence intervals in parameter estimation. For example, the $90 \%$ confidence region for the three-dimensional parameter space of $(M, \eta, \chi)$ for signals with SNR 10 is given by the region with matches above $\sim 0.97$. This allows us to estimate how the massratio-spin degeneracy will be reflected in parameter estimation. We show that for modest SNRs $(\sim 10)$ it may be difficult to determine whether a binary includes spin, and even for high SNRs $(\sim 30)$, the mass-ratio-spin degeneracy impairs the accurate recovery in the individual black-hole masses; see Figs. 9 and 10. These results will affect the astrophysical conclusions that can be drawn from GW observations, and this will be explored in more detail in future work. It would also be interesting to exploit our knowledge of the mass-ratio-spin degeneracy in a jump proposal for Bayesian parameter-estimation codes [15-17].

Our results are restricted to aligned-spin binaries and will be affected by the inclusion of precession effects. At present, no complete inspiral-merger-ringdown model for precessing systems exists. To date, there is no detailed study of the effects of precession on parameter degeneracy for stellar mass black holes, even with inspiral-only waveforms. It has, however, been shown that the inclusion of precession in supermassive black hole waveforms detectable by LISA can significantly improve the accuracy with which the mass ratio and component spins can be recovered $[39,40]$, although the degeneracy still persists at some level when the spins are close to aligned [41]. It is difficult to immediately translate these results to aLIGO, in large part due to the fact that the typical SNR of such systems would be around 1000, rather than 10 or 20 as discussed here. At high SNRs, even subdominant effects will be well measured and consequently break the observed degeneracy. It remains to be seen whether precession at moderate SNRs can alleviate the degeneracy we discuss and allow for accurate measurement of the component masses and spins. In general it is far more likely that increasing the dimensionality of the model parameter space will increase the parameter uncertainty, because the confidence regions will now correspond to lower matches. The recent results in Ref. [9] show that precession effects have only a weak impact on the phasing, which suggests that while the inclusion of precession is unlikely to break the degeneracy discussed here, it is also unlikely to introduce an additional degeneracy in these three parameters. On the other hand, the inclusion of higher harmonics, which were ignored in this study, may improve the accuracy of the parameters (as was seen for high SNR inspiral-only signals in both ground- [42] and space-based [41] detectors). The net impact of these two effects remains to be studied in more detail in future work.

\section{ACKNOWLEDGMENTS}

S.F. and M.H. were supported by Science and Technology Facilities Council Grants No. ST/H008438/1 and No. ST/I001085/1. S. F. also acknowledges the support of the Royal Society. We are particularly grateful to P. Ajith, Sascha Husa and Frank Ohme for sharing with us their implementations of the PhenomB and PhenomC waveform families and match-calculation code. We also thank Chris Messenger and John Veitch for useful discussions.
[1] J. Abadie et al. (LIGO Scientific), Classical Quantum Gravity 27, 173001 (2010).

[2] M. Dominik, K. Belczynski, C. Fryer, D. E. Holz, E. Berti, T. Bulik, I. Mandel, and R. O'Shaughnessy, Astrophys. J. 759, 52 (2012).

[3] F. Ohme, Classical Quantum Gravity 29, 124002 (2012).

[4] M. Hannam, Classical Quantum Gravity 26, 114001 (2009).
[5] P. Ajith et al., Classical Quantum Gravity 29, 124001 (2012).

[6] P. Ajith et al., Phys. Rev. Lett. 106, 241101 (2011).

[7] L. Santamaria et al., Phys. Rev. D 82, 064016 (2010).

[8] Y. Pan, A. Buonanno, L. T. Buchman, T. Chu, L.E. Kidder, H. P. Pfeiffer, and M. A. Scheel, Phys. Rev. D 81, 084041 (2010). 
[9] P. Schmidt, M. Hannam, and S. Husa, Phys. Rev. D 86, 104063 (2012).

[10] C. Cutler and E. E. Flanagan, Phys. Rev. D 49, 2658 (1994).

[11] E. Poisson and C. M. Will, Phys. Rev. D 52, 848 (1995).

[12] J. Abadie et al. (LIGO Scientific Collaboration and Virgo Collaboration), Phys. Rev. D 85, 082002 (2012).

[13] J. Abadie et al. (LIGO Scientific Collaboration and Virgo Collaboration), Phys. Rev. D 83, 122005 (2011).

[14] D. A. Brown, I. Harry, A. Lundgren, and A. H. Nitz, Phys. Rev. D 86, 084017 (2012).

[15] M. van der Sluys, V. Raymond, I. Mandel, C. Röver, N. Christensen, V. Kalogera, R. Meyer, and A. Vecchio, Classical Quantum Gravity 25, 184011 (2008).

[16] M. V. van der Sluys, C. Röver, A. Stroeer, V. Raymond, I. Mandel, N. Christensen, V. Kalogera, R. Meyer, and A. Vecchio, Astrophys. J. Lett. 688, L61 (2008).

[17] J. Veitch and A. Vecchio, Phys. Rev. D 81, 062003 (2010).

[18] F. Feroz, M. P. Hobson, and M. Bridges, Mon. Not. R. Astron. Soc. 398, 1601 (2009).

[19] LSC Algorithm Library software packages LAL, LALWRAPPER, and LALAPPS.

[20] P. Ajith et al., Classical Quantum Gravity 24, S689 (2007).

[21] P. Ajith et al., Phys. Rev. D 77, 104017 (2008); 79, 129901 (E) (2009).

[22] P. Ajith, Classical Quantum Gravity 25, 114033 (2008).

[23] P. Ajith et al., arXiv:0709.0093.

[24] B. Abbott et al. (LIGO Scientific), Rep. Prog. Phys. 72, 076901 (2009).

[25] D. Shoemaker (Advanced LIGO Team), Advanced LIGO Reference Design Report No. LIGO-M060056, 2009.
[26] G. M. Harry (LIGO Scientific Collaboration), Classical Quantum Gravity 27, 084006 (2010).

[27] D. Shoemaker and G. Losurdo, LIGO Project Technical Report No. LIGO-G1000176-v7, 2010.

[28] The LIGO Scientific Collaboration, Technical Report No. LIGO-T0900288-v3, 2009.

[29] C. Cutler and E. E. Flanagan, Phys. Rev. D 49, 2658 (1994).

[30] S. Babak, R. Balasubramanian, D. Churches, T. Cokelaer, and B.S. Sathyaprakash, Classical Quantum Gravity 23, 5477 (2006).

[31] S. Babak et al., arXiv:1208.3491 [Phys. Rev. D (to be published)].

[32] L. Lindblom, B. Owen, and D. Brown, Phys. Rev. D 78, 124020 (2008).

[33] P. Ajith, Phys. Rev. D 84, 084037 (2011).

[34] M. Boyle, D. A. Brown, and L. Pekowsky, Classical Quantum Gravity 26, 114006 (2009).

[35] B. Sathyaprakash and S. Dhurandhar, Phys. Rev. D 44, 3819 (1991).

[36] S. Droz, D. J. Knapp, E. Poisson, and B. J. Owen, Phys. Rev. D 59, 124016 (1999).

[37] M. Vallisneri, Phys. Rev. D 77, 42001 (2008).

[38] A. Buonanno, B. Iyer, E. Ochsner, Y. Pan, and B. Sathyaprakash, Phys. Rev. D 80, 084043 (2009).

[39] R. Lang and S. Hughes, Phys. Rev. D 74, 122001 (2006).

[40] A. Vecchio, Phys. Rev. D 70, 042001 (2004).

[41] R. Lang, S. Hughes, and N. Cornish, Phys. Rev. D 84, 022002 (2011).

[42] C. V.D. Broeck and A. S. Sengupta, Classical Quantum Gravity 24, 1089 (2007). 